Pacific Journal of Mathematics

ON THE ATOMIC DECOMPOSITION FOR HARDY SPACES 


\section{ON THE ATOMIC DECOMPOSITION FOR HARDY SPACES}

\section{J. Michael Wilson}

We give an extremely easy proof of the atomic decomposition for distributions in $H^{p}\left(\mathbf{R}_{+}^{n+1}\right), 0<p \leq 1$. Our proof uses only properties of the nontangential maximal function $u^{*}$. We then adapt our argument to give a "direct" proof of the Chang-Fefferman decomposition for $H^{p}\left(\mathbf{R}_{+}^{2} \times \mathbf{R}_{+}^{2}\right)$.

I. Introduction. Let $\mathbf{R}_{+}^{n+1}=\left\{(x, y): x \in \mathbf{R}^{n}, y>0\right\}$. For $u(x, y)$ harmonic on $\mathbf{R}_{+}^{n+1}$ and $A>0$ define

$$
u_{A}^{*}(x)=\sup _{|x-t|<A y}|u(t, y)|
$$

We say that $u \in H^{p}$ if $u_{A}^{*} \in L^{p}$, for any $A$, and set $\|u\|_{H^{p}}=\left\|u_{1}^{*}\right\|_{L^{p}}$. If $u \in H^{p}, 0<p<\infty$, then $f=\lim _{y \rightarrow 0} u(\cdot, y)$ exists (in $\mathscr{S}^{\prime}$ ) and is said to be in $H^{p}$. We set $\|f\|_{H^{p}}=\|u\|_{H^{p}}$ (see [6]).

For $0<p \leq 1$, a $p$-atom is a function $a(x) \in L^{2}\left(\mathbf{R}^{n}\right)$ satisfying:

$(\alpha)$ supp $a \subset Q, Q$ a cube.

( $\beta)\|a\|_{2} \leq|Q|^{1 / 2-1 / p}(|Q|=$ the volume of $Q)$.

( $\gamma$ ) $\int a(x) x^{\alpha} d x=0$ for all monomials $x^{\alpha}$ with $|\alpha| \leq\left[n\left(p^{-1}-1\right)\right]$.

The following theorem is well known [4] [7] [10]:

THEOREM A. Let $f \in H^{p}, 0<p \leq 1$. There exist $p$-atoms $a_{k}$ and numbers $\lambda_{k}$ such that

$$
f=\sum \lambda_{k} a_{k} \text { in } \mathscr{S}^{\prime}
$$

The $\lambda_{k}$ satisfy $\Sigma\left|\lambda_{k}\right|^{p} \leq C(p, n)\|f\|_{H^{p}}^{p}$. Conversely, every sum (1) satisfies

$$
\|f\|_{H^{p}}^{p} \leq C(p, n) \sum\left|\lambda_{k}\right|^{p}
$$

Now let $u$ be biharmonic on $\mathbf{R}_{+}^{2} \times \mathbf{R}_{+}^{2}$. Define

$$
u_{A}^{*}\left(x_{1}, x_{2}\right)=\sup _{\substack{\left|x_{i}-t_{i}\right|<A y_{i} \\ i=1,2}}\left|u\left(t_{1}, y_{1}, t_{2}, y_{2}\right)\right|
$$


As before, we say that $u \in H^{p}\left(\mathbf{R}_{+}^{2} \times \mathbf{R}_{+}^{2}\right)$ if $u_{A}^{*} \in L^{p}\left(\mathbf{R}^{2}\right)$, and we set $\|u\|_{H^{p}}=\left\|u_{1}^{*}\right\|_{L^{p}}$. Such $u$ give rise to boundary distributions $f$, which are said to be in $H^{p}$. (See [2].)

For $0<p \leq 1$, a Chang-Fefferman $p$-atom is a function $a \in L^{2}\left(\mathbf{R}^{2}\right)$ satisfying:

$\left(\alpha^{\prime}\right)$ supp $a \subset \Omega, \Omega$ open, $|\Omega|<\infty$.

$\left(\beta^{\prime}\right)\|a\|_{2} \leq|\Omega|^{1 / 2-1 / p}$.

$\left(\gamma^{\prime}\right) a=\sum_{R} \lambda_{R} a_{R}$, where $\lambda_{R}$ are numbers and the $a_{R}$ are functions (called “elementary particles") satisfying:

(i) $\operatorname{supp} a_{R} \subset \tilde{R} \subset \Omega$ where $R=I \times J, I, J$ dyadic intervals, and $\tilde{R}$ denotes the triple of $R$.

(ii)

$$
\left\|\frac{\partial^{L} a_{R}}{\partial x_{1}^{L}}\right\|_{\infty} \leq \frac{1}{\sqrt{|R|}|I|^{L}} \text { and }\left\|\frac{\partial^{L} a_{R}}{\partial x_{2}^{L}}\right\|_{\infty} \leq \frac{1}{\sqrt{|R|}|J|^{L}}
$$

for all $L \leq[2 / p-1 / 2]$

(iii)

$$
\int a\left(\tilde{x}_{1}, x_{2}\right) x_{2}^{k} d x_{2}=0 \text { and } \int a\left(x_{1}, \tilde{x}_{2}\right) x_{1}^{k} d x_{1}=0
$$

for all $\left(\tilde{x}_{1}, \tilde{x}_{2}\right) \in \mathbf{R}^{2}$ and all $k \leq[2 / p-3 / 2]$. And

$$
\left(\sum_{R} \lambda_{R}^{2}\right)^{1 / 2} \leq|\Omega|^{1 / 2-1 / p}
$$

If the "atoms" are Chang-Fefferman atoms, then Theorem $\mathrm{A}$ is true for $f \in H^{p}\left(\mathbf{R}_{+}^{2} \times \mathbf{R}_{+}^{2}\right)$ [2] [3].

Until now, proofs of the atomic decomposition have relied on showing that $u^{*} \in L^{p}$ implies that some auxiliary function (such as the "grand" maximal function or the $S$-function) is in $L^{p}$. In this paper, we give proofs which get the atoms directly from " $u^{*} \in L^{p}$ ".

REMARK. Our argument is somewhat like that of A. P. Calderón in [1]. Calderón's " $u^{*}$ " is the sum of two real-variable maximal functions. He writes his reproducing formula (see below) in terms of one kernel, and uses the other kernel to control the $L^{\infty}$ size of his atoms. Our proof uses Green's Theorem to get $L^{2}$ bounds. This approach lets us adapt our proof to the bidisc setting, where $L^{\infty}$ atoms do not seem to be the "right" ones.

II. The case $H^{p}\left(\mathbf{R}_{+}^{2}\right)$. Let $\psi \in C^{\infty}(\mathbf{R})$ be real, radial, supp $\psi \subset\{|x|$ $\leq 1\}, \psi$ has the cancellation property $\gamma$ ), and

$$
\int_{0}^{\infty} e^{-\theta} \hat{\psi}(\theta) d \theta=-1 .
$$

For $y>0$, set $y^{-1} \psi(t / y)=\psi_{y}(t)$. 
Take $f \in L^{2} \cap H^{p}, f$ real-valued, $u=P_{y} * f$ (the Poisson integral of $f)$. By Fourier transforms

$$
f=\int_{\mathbf{R}_{+}^{2}} \frac{\partial u}{\partial y}(t, y) \psi_{y}(x-t) d t d y \quad \text { in } \mathscr{S}^{\prime} .
$$

(This trick is due to A. P. Calderón.) For $k=0, \pm 1, \pm 2, \ldots$, define

$$
E^{k}=\left\{u_{2}^{*}>2^{k}\right\}=\bigcup_{j=1}^{\infty} I_{j}^{k}
$$

where the $I_{j}^{k}$ are component intervals. For $I$ an interval, let

$$
\hat{I}=\left\{(t, y) \in \mathbf{R}_{+}^{2}:(t-y, t+y) \subset I\right\}
$$

be the "tent" region. Define $\hat{E}^{k}=\bigcup \hat{I}_{j}^{k}, T_{j}^{k}=\hat{I}_{j}^{k} \backslash \hat{E}^{k+1}$. Then

$$
f=\sum_{k, j} \int_{T_{j}^{k}} \frac{\partial u}{\partial y}(t, y) \psi_{y}(x-t) d t d y=\sum_{k, j} g_{j}^{k}=\sum_{k, j} \lambda_{j}^{k} a_{j}^{k},
$$

where $\lambda_{j}^{k}=C 2^{k}\left|I_{j}^{k}\right|^{1 / p}$ and the $a_{j}^{k}$ (we claim) are atoms. The $a_{j}^{k}$ inherit $\gamma$ from $\psi$, and obviously supp $a_{j}^{k} \subset \tilde{I}_{j}^{k}$. Note also that

$$
\sum\left(\lambda_{j}^{k}\right)^{p} \leq C \int\left(u_{2}^{*}\right)^{p} d x \leq C\|u\|_{H^{p}}^{p}
$$

Thus, we are done if we can show

$$
\left\|g_{j}^{k}\right\|_{2} \leq C 2^{k}\left|I_{j}^{k}\right|^{1 / 2}
$$

We do this by duality. Let $h \in L^{2}(\mathbf{R}),\|h\|_{2}=1$. Then

$$
\begin{aligned}
\left|\int h(x) g_{j}^{k}(x) d x\right| & =\left|\int_{T^{k}} \frac{\partial u}{\partial y}(t, y)\left(h * \psi_{y}(t)\right) d t d y\right| \\
& \leq\left(\int_{T_{j}^{k}} y|\nabla u|^{2} d t d y\right)^{1 / 2}\left(\int_{\mathbf{R}_{+}^{2}}\left|h * \psi_{y}(t)\right|^{2} \frac{d t d y}{y}\right)^{1 / 2}
\end{aligned}
$$

(Plancherel)

$$
\leq C\left(\int_{T_{J}^{k}} y|\nabla u|^{2} d t d y\right)^{1 / 2}
$$

We estimate the last integral by Green's Theorem. It is bounded by

$$
\left(\int_{\partial T_{j}^{k}}\left(|u| y\left|\frac{\partial u}{\partial \nu}\right|+\frac{1}{2} u^{2}\left|\frac{\partial y}{\partial \nu}\right|\right) d s\right)
$$

( $\partial / \partial \nu$ is outward normal; $\partial T_{j}^{k}$ is just smooth enough to let us use Green's Theorem). Because of the " 2 " (in $u_{2}^{*}$ ), both $|u|$ and $y|\nabla u|$ are bounded by $C 2^{k}$ on $\partial T_{j}^{k}$. Since $|\partial y / \partial \nu| \leq 1$ and $\left|\partial T_{j}^{k}\right| \leq C\left|I_{j}^{k}\right|$, the last term is no larger than $C 2^{k}\left|I_{j}^{k}\right|^{1 / 2}$. 
III. The case $H^{p}\left(\mathbf{R}_{+}^{n+1}\right)$. Let $\psi$ be as in II, except now $\psi \in C^{\infty}\left(\mathbf{R}^{n}\right)$. Let $f \in H^{p} \cap L^{2}$ and $u$ be as before. Define

$$
E^{k}=\left\{u_{10^{n}}^{*}>2^{k}\right\}=\bigcup_{j=1}^{\infty} \Omega_{j}^{k} ;
$$

where the $\Omega_{j}^{k}$ are Whitney cubes (for the definition see [9], p. 167). For $\Omega$ a cube in $\mathbf{R}^{n}$, define

$$
\hat{\Omega}=\{(t, y): t \in \Omega, 0<y<l(\Omega)\}
$$

where $l(\Omega)=$ sidelength of $\Omega$. Define

$$
\hat{E}^{k}=\bigcup \hat{\Omega}_{j}^{k}, \quad T_{j}^{k}=\hat{\Omega}_{j}^{k} \backslash \hat{E}^{k+1} .
$$

With these modifications, the preceding argument goes over practically verbatim; the details are left to the reader.

IV. The case $H^{p}\left(\mathbf{R}_{+}^{2} \times \mathbf{R}_{+}^{2}\right)$. We first show that the proof in II yields a Chang-Fefferman decomposition for $\mathbf{R}_{+}^{2}$. For $I \subset \mathbf{R}$ a dyadic interval, let

$$
I^{+}=\{(t, y): t \in I,|I| / 2<y \leq|I|\}
$$

Define

$$
\begin{aligned}
\mathscr{I}_{j}^{k} & =\left\{Q=I^{+} \cap T_{j}^{k}\right\} \\
g_{Q} & =\int_{Q} \frac{\partial u}{\partial y}(t, y) \psi_{y}(x-t) d t d y=\lambda_{j}^{k} \lambda_{Q} a_{Q} \quad \text { for } Q \in \mathscr{I}_{j}^{k},
\end{aligned}
$$

where we set

$$
\lambda_{Q}=C\left(\lambda_{j}^{k}\right)^{-1}\left(\int_{Q} y|\nabla u|^{2} d t d y\right)^{1 / 2} .
$$

Then it is easily verified that the $a_{Q}$ have the right cancellation, support and smoothness properties for elementary particles. And obviously

$$
\begin{aligned}
& a_{j}^{k}=\sum_{Q \in \mathscr{F}_{j}^{k}} \lambda_{Q} a_{Q}, \\
& \left(\sum_{Q \in \mathscr{I}_{J}^{k}} \lambda_{Q}^{2}\right)^{1 / 2} \leq\left|\tilde{I}_{j}^{k}\right|^{1 / 2-1 / p} .
\end{aligned}
$$

In order to do our proof in $\mathbf{R}_{+}^{2} \times \mathbf{R}_{+}^{2}$, we need tents, and we need a way to do Green's Theorem. For these, we need some notation. 
For $(t, y)=\left(t_{1}, y_{1}, t_{2}, y_{2}\right) \in\left(\mathbf{R}_{+}^{2}\right)^{2}$, let $R_{t, y}$ be the rectangle with sides parallel to the coordinate axes, centered at $\left(t_{1}, t_{2}\right) \in \mathbf{R}^{2}$, and with dimensions $2 y_{1} \times 2 y_{2}$.

Take $f \in L^{2} \cap H^{p}, u=P_{y_{1}} \cdot P_{y_{2}} * f$ (the double Poisson integral of $f$ ). Let $\psi$ be as in II but with cancellation corresponding to (iii). Then

$$
f=\int_{\left(\mathbf{R}_{+}^{2}\right)^{2}} \frac{\partial^{2} u}{\partial y_{1} \partial y_{2}}(t, y) \psi_{y_{1}}\left(x_{1}-t_{1}\right) \psi_{y_{2}}\left(x_{2}-t_{2}\right) d t d y \quad \text { in } \mathscr{S}^{\prime} .
$$

Let $M$ be the strong maximal function. Let $\varepsilon>0$ be small, to be chosen later. Define

$$
E^{k}=\left\{u_{100}^{*}>2^{k}\right\}, \quad F^{k}=\left\{M \chi_{E^{k}}>\varepsilon\right\}
$$

It is a fact that $\left|F^{k}\right| \leq C_{\varepsilon}\left|E^{k}\right|$. Set

$$
\begin{aligned}
\hat{F}^{k} & =\left\{(t, y): R_{t, y} \subset F^{k}\right\}, \\
T^{k} & =\hat{F}^{k} \backslash \hat{F}^{k+1}, \\
g^{k} & =\int_{T^{k}} \frac{\partial^{2} u}{\partial y_{1} \partial y_{2}}(t, y) \psi_{y_{1}}\left(x_{1}-t_{1}\right) \psi_{y_{2}}\left(x_{2}-t_{2}\right) d t d y=\lambda_{k} a_{k},
\end{aligned}
$$

where we set $\lambda_{k}=C 2^{k}\left|E^{k}\right|^{1 / p}$.

For $R=I \times J, I, J$ dyadic intervals, let $R^{+}=I^{+} \times J^{+} \subset R_{+}^{2} \times R_{+}^{2}$. Set

$$
\begin{aligned}
\mathscr{I}_{k} & =\left\{Q=R^{+} \cap T^{k}\right\}, \\
g_{Q} & =\int_{Q} \frac{\partial^{2} u}{\partial y_{1} \partial y_{2}}(t, y) \psi_{y_{1}}\left(x_{1}-t_{1}\right) \psi_{y_{2}}\left(x_{2}-t_{2}\right) d t d y \\
& =\lambda_{k} \lambda_{Q} a_{Q} \quad\left(Q \in \mathscr{I}^{k}\right),
\end{aligned}
$$

where we set

$$
\lambda_{Q}=C\left(\lambda_{k}^{-1}\right)\left(\int_{Q} y_{1} y_{2}\left|\nabla_{1} \nabla_{2} u\right|^{2} d t d y\right)^{1 / 2}
$$

with

$$
\left|\nabla_{1} \nabla_{2} u\right|^{2}=\left|\frac{\partial^{2} u}{\partial x_{1} \partial x_{2}}\right|^{2}+\left|\frac{\partial^{2} u}{\partial x_{1} \partial y_{2}}\right|^{2}+\left|\frac{\partial^{2} u}{\partial y_{1}, \partial x_{2}}\right|^{2}+\left|\frac{\partial^{2} u}{\partial y_{1} \partial y_{2}}\right|^{2} .
$$
show

Then, in exact analogy to case II, everything will be done once we

$$
\int_{T^{k}} y_{1} y_{2}\left|\nabla_{1} \nabla_{2} u\right|^{2} d t d y \leq C 2^{2 k}\left|E^{k}\right|
$$


For this we need a lemma of Merryfield. The lemma requires a little more notation.

Let $\eta \in C^{\infty}(\mathbf{R}), \eta \geq 0, \operatorname{supp} \eta \subset[-1,1], \eta \geq \frac{1}{2}$ on $\left[-\frac{1}{2}, \frac{1}{2}\right]$ and $\int \eta$ $=1$. Define

$$
\Phi_{y_{1} y_{2}}\left(t_{1}, t_{2}\right)=\eta_{y_{1}}\left(t_{1}\right) \cdot \eta_{y_{2}}\left(t_{2}\right) .
$$

For $E \subset \mathbf{R}^{2}$, set

$$
V_{E}(t, y)=\Phi_{y} * \chi_{E}(t), \quad(t, y) \in\left(\mathbf{R}_{+}^{2}\right)^{2} .
$$

Now, $V_{E}(t, y)$ is essentially the density of $E$ in $R_{t, y}$. In particular, if this density is greater than $1-\varepsilon, \varepsilon$ small, then $V_{E}(t, y)>10^{-6}$.

Merryfield's lemma is [8]:

Lemma. Let $u \in H^{p}\left(\mathbf{R}_{+}^{2} \times \mathbf{R}_{+}^{2}\right), p<2$, and let $u_{100}^{*} \leq \lambda$ on $E \subset \mathbf{R}^{2}$. Then

$$
\int_{\left(\mathbf{R}_{+}^{2}\right)^{2}} y_{1} y_{2}\left|\nabla_{1} \nabla_{2} u\right|^{2} V_{E}^{2}(t, y) d t d y \leq C \lambda^{2}|E|
$$

(Note: Merryfield states this for $E$ open, but openness, as his proof shows, is not required.)

Let us set $G^{k}=F^{k} \backslash E^{k+1}$. Merryfield's lemma says that

$$
\int_{\mathbf{R}_{+}^{2}} y_{1} y_{2}\left|\nabla_{1} \nabla_{2} u\right|^{2} V_{G^{k}}^{2}(t, y) d t d y \leq C 2^{2 k}\left|G^{k}\right| \leq C 2^{2 k}\left|E^{k}\right|
$$

Therefore, we will have (2) (and be done) if we can show

$$
V_{G^{k}}>10^{-6} \text { on } T^{k} .
$$

Take $(t, y) \in T^{k}$. Then $R_{t, y} \subset F^{k}$ but $R_{t, y} \not \subset F^{k+1}$. So there is an $x \in R_{t, y} \cap\left(F^{k} \backslash F^{k+1}\right)$. Since $x \notin F^{k+1}, M \chi_{E_{1}^{k+}}(x) \leq \varepsilon$. From the definition of $M$, this implies

$$
\left|R_{t, y} \cap E^{k+1}\right| /\left|R_{t, y}\right| \leq \varepsilon .
$$

Since $R_{t, y} \subset F^{k}$,

$$
\left|R_{t, y} \cap\left(F^{k} \backslash E^{k+1}\right)\right| /\left|R_{t, y}\right| \geq 1-\varepsilon .
$$

But $F^{k} \backslash E^{k+1}=G^{k}$, and this implies that $V_{G^{k}}(t, y)>10^{-6}$, for $\varepsilon$ small. $\square$ 


\section{REFERENCES}

[1] A. P. Calderón, An atomic decomposition of distributions in parabolic $H^{p}$ spaces, Adv. in Math., 25 (1977), 216-225.

[2] S.-Y. Chang and R. Fefferman, $A$ continuous version of duality of $H^{1}$ with $B M O$ on the bidisc, Ann. of Math., 112 (1980), 179-201.

[3] The Calderon-Zygmund decomposition on product domains, preprint.

[4] R. R. Coifman, A real variable characterization of $H^{p}$, Studia Math., 51 (1974), 269-74.

[5] R. Coifman, Y. Meyer and E. M. Stein, Un nouvel espace fonctionnel adapté à l'étude des opérateurs définie par des intégrales singulieres, preprint.

[6] C. Fefferman and E. M. Stein, $H^{p}$ spaces of several variables, Acta Math., 129 (1972), 137-193.

[7] R. H. Latter, $A$ characterization of $H^{p}\left(\mathbf{R}^{p}\right)$ in terms of atoms, Studia Math., 62 (1978), 93-101.

[8] K. G. Merryfield, $H^{p}$ spaces in poly-half spaces, Ph.D. Thesis, University of Chicago, 1980.

[9] E. M. Stein, Singular Integrals and Differentiability Properties of Functions, Princeton University Press, 1970.

[10] J. M. Wilson, A simple proof of the atomic decomposition for $H^{p}\left(\mathbf{R}^{n}\right), 0<p \leq 1$, Studia Math., 74 (1982), 25-33.

Received April 26, 1983 and in revised form July 8, 1983. Partially supported under NSF Grant MCS 8203319.

UNIVERSITY OF CHICAGO

CHICAgo, IL 60637 



\section{PACIFIC JOURNAL OF MATHEMATICS \\ EDITORS}

Donald BABBITT (Managing Editor)

University of California

Los Angeles, CA 90024

J. Dugundu

University of Southern Californa

Los Angeles, CA 90089-1113

R. FINN

Stanford University

Stanford, CA 94305

HermanN FLaSChKa

University of Arizona

Tucson, AZ 85721

\author{
C. C. MOORE \\ University of California \\ Berkeley, CA 94720 \\ ARTHur Ogus \\ University of California \\ Berkeley, CA 94720 \\ Hugo Rossi \\ University of Utah \\ Salt Lake City, UT 84112 \\ H. SAMELSON \\ Stanford University \\ Stanford, CA 94305
}

ASSOCIATE EDITORS
R. ARENS
E. F. BECKENBACH
B. H. NeUmanN
F. WOLF
K. YOSHIDA (1906-1982)

\section{SUPPORTING INSTITUTIONS}

UNIVERSITY OF ARIZONA

UNIVERSITY OF BRITISH COLUMBIA

UNIVERSITY OF OREGON

CALIFORNIA INSTITUTE OF TECHNOLOGY

UNIVERSITY OF CALIFORNIA

MONTANA STATE UNIVERSITY

UNIVERSITY OF SOUTHERN CALIFORNIA

UNIVERSITY OF NEVADA, RENO

STANFORD UNIVERSITY

UNIVERSITY OF HAWAII

NEW MEXICO STATE UNIVERSITY

UNIVERSITY OF TOKYO

UNIVERSITY OF UTAH

WASHINGTON STATE UNIVERSITY

OREGON STATE UNIVERSITY

UNIVERSITY OF WASHINGTON 


\section{Pacific Journal of Mathematics}

\section{Vol. 116, No. $1 \quad$ November, 1985}

K. Adachi, Le problème de Lévi pour les fibrés grassmanniens et les variétés

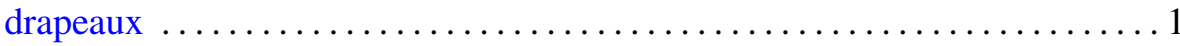

John MacLeod Ball, Remarks on the paper: "Basic calculus of variations" . . . 7 John Kelly Beem and Phillip E. Parker, Whitney stability of solvability . . . 11 Alberto Facchini, Decompositions of algebraically compact modules .......25

S. S. Khare, Finite group action and equivariant bordism $\ldots \ldots \ldots \ldots . \ldots 39$

Horst Leptin, A new kind of eigenfunction expansions on groups $\ldots \ldots \ldots . .45$

Pei-Kee Lin, Unconditional bases and fixed points of nonexpansive

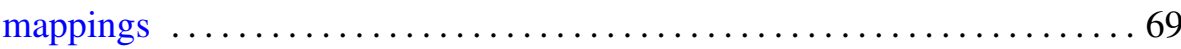

Charles Livingston, Stably irreducible surfaces in $S^{4} \ldots \ldots \ldots \ldots \ldots 77$

Kevin Mor McCrimmon, Nonassociative algebras with scalar involution . . .885

Albert Milani, Singular limits of quasilinear hyperbolic systems in a

bounded domain of $\mathbf{R}^{3}$ with applications to Maxwell's equations

Takemi Mizokami, On $M$-structures and strongly regularly stratifiable

spaces

Jesper M. Møller, On the homology of spaces of sections of complex

projective bundles

Nikolaos S. Papageorgiou, Carathéodory convex integrand operators and

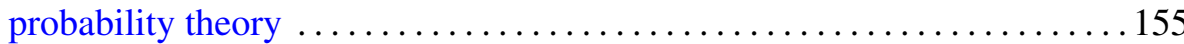

Robert John Piacenza, Transfer in generalized prestack cohomology 185

Lance W. Small and Adrian R. Wadsworth, Integrality of subrings of matrix rings ...

James Michael Wilson, On the atomic decomposition for Hardy spaces 